Modeling partially incomplete marital satisfaction data

Peer-reviewed author version

JANSEN, Ivy; Van den Troost, Ann; MOLENBERGHS, Geert; Vermulst, Ad A. \&

Gerris, Jan R.M. (2006) Modeling partially incomplete marital satisfaction data. In:

SOCIOLOGICAL METHODS \& RESEARCH, 35(1). p. 113-136.

DOI: $10.1177 / 0049124106289163$

Handle: http://hdl.handle.net/1942/1970 


\section{Modeling Partially Incomplete Marital Satisfaction Data}

\section{Ivy Jansen, ${ }^{1}$ Ann Van den Troost,${ }^{2}$ Geert Molenberghs, ${ }^{3}$ Ad A. Vermulst ${ }^{4}$ and Jan R.M. Gerris ${ }^{5}$.}

${ }^{1}$ Center for Statistics, Hasselt University, Agoralaan gebouw D, 3590 Diepenbeek, Belgium. Email: ivy.jansen@uhasselt.be Phone: +3211268233Ｆax: +321126 8299

${ }^{2}$ Katholieke Universiteit Leuven, Faculteit Sociale Wetenschappen, Departement Sociologie, Centrum voor Bevolkings- en Gezinsonderzoek, E. Van Evenstraat 2b, 3000 Leuven, Belgium. Email: ann.vandentroost@soc.kuleuven.be～Phone: +32 $16323177 \quad$ Fax: +32 163233 65

${ }^{3}$ Center for Statistics, Hasselt University, Agoralaan gebouw D, 3590 Diepenbeek, Belgium. Email: geert.molenberghs@uhasselt.be Phone: +32112682 38 Fax: +3211268299

${ }^{4}$ Institute of Family and Child Care Studies, Radboud University Nijmegen, P.O. Box 9104, 6500 HE Nijmegen, the Netherlands. Email: a.vermulst@home.nl Phone: +31 24361 6283 Fax: +312436127 76

${ }^{5}$ Institute of Family and Child Care Studies, Radboud University Nijmegen, P.O. Box 9104, 6500 HE Nijmegen, the Netherlands. $\quad$ Email: j.gerris@ped.kun.nl Phone: +31 2436121 23 Fax: +312436127 76

Key words: Complete Case Analysis; Direct Likelihood; Dropout; Imputation; Missing at Random; Missing Completely at Random; Missing Data. 


\section{Biographical sketch}

- Ivy Jansen is M.Sc. in Biostatistics, and defended her Ph.D. in Biostatistics on June 24, 2005 at the Center for Statistics, Hasselt University. Her research interests focus on categorical longitudinal data, missing data, and sensitivity analysis tools. Now she is working as a post-doctoral researcher on Bioinformatics.

- Ann Van den Troost, Ph.D. is researcher at the Center of Population and Family Research (Center of Sociological Research (CESO) - Catholic University of Leuven). Her sociological research activities and published work address family issues and marital satisfaction.

- Geert Molenberghs is Professor in Biostatistics and Head of Department at Hasselt University in Belgium. He published methodological work, including books, on longitudinal data, repeated categorical data and on the analysis of nonresponse in clinical and epidemiological studies. He is past Editor of Applied Statistics and Fellow of the American Statistical Association. He is President of the International Biometric Society (2004-2005).

- Ad Vermulst received his Ph.D. in the Social Sciences from the University of Groningen, the Netherlands. He is a senior researcher in family studies at the University of Nijmegen, the Netherlands. His research and publications are in the field of personality, workstress, family stress, child rearing and smoke and drinking behavior of adolescents. His interest lies in statistical methodology like structural equation modeling.

- Jan Gerris is full professor in Family Research at the Radboud University Nijmegen (the Netherlands). His current research is focused on the role of personality in family 
relations and parenting. He was and still is supervising different research projects on family relations, e.g. the longitudinal project of Parenting and Family Relationships in the Netherlands, which started in 1990 (first wave). He is president and co-founder of the European Society on Family Relations (ESFR). He is editorial board member and reviewer of various international journals.

\begin{abstract}
We analyze data on marital satisfaction, obtained from couples at two distinct moments in time $(1990,1995)$. The data are of a bivariate longitudinal type. Moreover, some couples provide incomplete records only, usually because the 1995 follow-up interview has not taken place. We propose a hierarchical modeling strategy which takes all of these features into account and is more generally valid than a classical complete case or single imputation-based strategy.
\end{abstract}




\section{Introduction}

Understanding the nature and the determinants of change in marital quality is one of the most crucial issues in contemporary marital research. Therefore, the use of longitudinal data in general and panel data in particular is considered as an important source of theory development in this field (Karney and Bradbury, 1995). However, the advantages of panel data are often reduced by the loss of panel members in follow-up surveys. The risk that this panel attrition is selective and not at random, may call into question the validity of the available data. This study examines the demographic correlates of attrition to gain insight in the extent to which demographic differences between panel members included and those lost to follow up possibly may affect the responses on marital quality.

Drawing on a sample of long-term married couples, we attempt to gain insight into the extent to which marital quality is subject to change as a consequence of spouses' position in the social structure. For example, there is evidence that individuals who are lower educated or have less material resources report lower marital satisfaction (Lewis and Spanier, 1979). Research also suggests that marital satisfaction is lower in longer-term marriages than in those of short duration (Johnson et al., 1992). This might be due to an increasing disenchantment after the first years of marriage. Moreover, evidence is obtained for a lower marital quality reported by individuals who married at a young age (Holman, 2001). The reason is that these spouses should be less prepared to fulfill their marital roles adequately. In a similar vein, Amato (1996) asserts that individuals confronted with the divorce of their parents and the inherently conflictual family climate associated with it, have a higher risk to develop less satisfying and unstable marriages themselves. Besides, children may also put burden on a marital relationship. The more children present, the higher the parental demands are and the more likely this results in negative marital 
outcomes (Lavee et al., 1996; Rogers and White, 1998).

However, the question as to whether the aforementioned variables also affect marital quality change in long-term marriages is not addressed. From a family life cycle perspective this insight may be particularly relevant. According to this perspective families have different needs and orientations at different stages of the life cycle. These stage-specific demands should explain the U-shaped pattern of marital quality over the marital career, as was once believed. Yet, since the early nineties it became clear that marital quality probably does not follow a U-shaped curve, but instead gradually declines over the marital career (Vaillant and Vaillant, 1993). This has led authors to question the importance of spousal background variables in this decline.

Moreover, and of major importance for the current paper, a challenge when analyzing data from studies of the type described above is the incompleteness of the outcomes due to dropout. In our case, incompleteness arises in part due to divorce during the study. Oftentimes, researchers opt for a so-called complete case analysis (Little and Rubin, 2002, $\mathrm{CC}$, ) restricting the analysis to couples with all responses obtained. While simple to perform, there is a strong danger for bias. In addition, since data on incomplete records are deleted, the statistical efficiency is reduced, leading to larger standard errors. These and other dangers of a $\mathrm{CC}$ analysis have been reported in a number of statistical application areas (Molenberghs et al., 2004; Jansen et al., 2005; Little, 1982, 1995). Alternatively, filling-in of incomplete data has been popular for a long time. These so-called single imputation methods are also described and discussed by Little and Rubin (2002) and carry even more problems than do CC methods. This is explicitly not true for the broadly valid and flexible multiple-imputation strategy, which is, together with the expectation-maximization algorithm, a viable alternative for direct likelihood. Especially 
when data are measured repeatedly, not only means and differences between groups, but also within-unit correlation and other modeling aspects tend to be distorted in non-trivial ways. CC and imputation methods require, at least, that missing data are missing completely at random (MCAR), a term coined by Rubin (1976), indicating that the occurrence of missingness is independent of both observed and unobserved outcomes. This is in contrast to the much weaker condition of MAR (missing at random), where missingness is allowed to depend on observed outcomes but, given these, not on unobserved ones. A key result of Rubin (1976) is that likelihood-based and Bayesian inferential methods are valid, as soon as missingness is MAR, without the need for formulating an explicit missing data model. In other words, under this assumption, one only requires a method and a corresponding software tool, that allows to use incomplete records alongside the complete ones. For this reason, such an approach is often called an ignorable likelihood-based (or Bayesian) analysis. Technically, one only requires software that can handle incomplete longitudinal sequences, such as the SAS procedure MIXED. Apart from so-called direct likelihood, multiple imputation and the expectation-maximization method are valid method within this category (Little and Rubin, 2002).

A few cautionary remarks are warranted. First, when at least part of the scientific interest is directed towards the nonresponse process (e.g., to study reasons for non-response), obviously both processes need to be considered. Under MAR, both processes can be modeled and parameters estimated separately. Secondly, it may be hard to rule out the operation of an MNAR mechanism, where missingness depends on unobserved outcomes, in addition to the observed ones. A full treatment of this category lies outside of the scope of this paper. Details about MNAR modelling and sensitivity analysis associated with it can be found in Verbeke and Molenberghs (2000). 
A formal data and modeling framework will be sketched in Section 3. Methods to deal with incomplete data are presented in Section 4, while the analysis of the case study, described in Section 2, is given in Section 5.

\section{Description of the Data}

The research sample consists of married men and women participating in the longitudinal research project "Child-rearing and family in the Netherlands". In 1990 and 1995 the same family members (wife, husband and target child) provided information about similar sets of measures. Families were recruited using a multi-stage sampling method. In a first stage, a sample was taken of all Dutch municipalities distinguished by regional zone and degree of urbanization. In a second stage, a sample of children aged 9 to 16 years old was taken in the selected municipalities. The children were selected in such a way that in each city as much boys as girls and as much children aged 9 to 12 as aged 13 to 16 were chosen. In 1990 this procedure resulted in a sample of 1829 families. The response ratio was $43 \%$ $(N=788)$. Families who refused to participate were asked for their reasons. The reasons are presented in Table 1.

Table 1 about here

Despite the reduction of the number of families, the sample was representative regarding regional zone and degree of urbanization. Of the 656 families who agreed in 1990 to participate in the second wave, 627 could be traced five years later. Of these contacted families, $484(77 \%)$ actually did participate in 1995 . The reasons for refusal are presented 
in Table 1. This sample proved to be still representative for regional zone but not for degree of urbanization. It appeared that primarily participants from the bigger cities refused to participate for the second time in the research project. More technical details on the database can be found in Gerris et al. $(1992,1993,1998)$. The data were gathered by means of structured interviews and questionnaires, completed by both the child and the parents. In order to establish a homogeneous research group for a study on marital quality, only first marriages in which both men and women have a Dutch nationality were selected. This selection resulted in a research group of 646 couples in 1990 and 386 couples in 1995.

Now we will give some more details about the responses of interest to measure marital quality, and about the covariates that will be taken into account because of potential influence on marital quality, as they were already mentioned in Section 1.

\subsection{Outcomes of Interest}

Marital satisfaction is measured by the Marital Satisfaction scale of Gerris et al. (1992, 1993, 1998). For formulating the items, satisfaction with the relationship and/or the partner was used as the guiding principle (e.g., "Generally, I'm dissatisfied with the relationship with my partner" or "If I could choose again, I would choose the same partner"). The scale consists of seven 7-point Likert items, ranging from $1=$ "do not agree" to $7=$ "completely agree".

The negative communication scale of Gerris et al. $(1992,1993)$ maps out the negative communication styles of the couple. Respondents were asked to indicate to what degree certain forms of negative communication are characteristic to their marital relationship (e.g., "My partner often blames me when we are quarrelling" or "My partner and I 
interrupt each other a lot when we are talking together"). The scale consists of six 7-point Likert items.

The open communication scale of Gerris et al. $(1992,1993)$ maps out the open communicational styles of the couple. Respondents were asked to indicate to what degree personal feelings and experiences were shared (e.g., "I often talk to my partner about things we are both interested in" or "I often talk to my partner about personal problems"). The open communication scale also consists of three 7-point Likert items The uniqueness and stability of thes concepts was demonstrated in Van den Troost et al. (2001).

\subsection{Covariates of Interest}

Education was measured in response to the question "What is your highest educational level?", using a nine-level scale ranging from $1=$ "elementary school" to $9=$ "university education". Family income was measured in response to the question "What is the monthly net family income (in guilders, approx. 0.45 euro)?" Seven income groups were distinguished: $1=$ "1100-1600", then in groups of 500 guilders, until $7=$ "more than 4500". Further covariates are year of birth, year of marriage, number of children and marital status of the parents of the couple. For the latter, to both husband and wife it was asked "The time you were living with your parents, they were married all the time?". Response categories were $1=$ "yes", $2=$ "no" and $3=$ "not applicable".

When data are incomplete, properly accounting for covariates will not only enhance the descriptive power of an outcome model, it may also reinforce the basis for MAR, thus validating direct likelihood and other MAR methods. Indeed, it is possible that an otherwise MNAR mechanism shifts to MAR or even MCAR by properly including 
covariates. At the same time, covariates may be prone to incompleteness, exactly as outcome variables. It is in such a case that multiple imputation is particularly useful (Little and Rubin, 2002), since the method allows for incomplete outcomes and covariates at the same time, while often direct likelihood poses challenges in this particular situation.

\section{Data Setting and Modeling Framework}

Assume that for subject $i=1, \ldots, N$ in the study a sequence of responses $Y_{i j}$ is designed to be measured at occasions $j=1, \ldots, n$. The outcomes are grouped into a vector $\boldsymbol{Y}_{i}=\left(Y_{i 1}, \ldots, Y_{i n}\right)^{\prime}$. In addition, define a dropout indicator $D_{i}$ for the occasion at which dropout occurs and adopt the convention that $D_{i}=n+1$ for a complete sequence. It is often convenient to split the vector $\boldsymbol{Y}_{i}$ into observed $\left(\boldsymbol{Y}_{i}^{o}\right)$ and missing $\left(\boldsymbol{Y}_{i}^{m}\right)$ components respectively.

In principle, one would like to consider the density of the full data $f\left(\boldsymbol{y}_{i}, d_{i} \mid \boldsymbol{\theta}, \boldsymbol{\psi}\right)$, where the parameter vectors $\boldsymbol{\theta}$ and $\boldsymbol{\psi}$ describe the measurement and missingness processes, respectively. Covariates are assumed to be measured, but have been suppressed from notation for simplicity.

Most strategies used to analyze such data are, implicitly or explicitly, based on two choices.

Model for measurements. A choice has to be made regarding the modeling approach to the measurements. Several views are possible.

View 1. One can choose to analyze the entire profile of outcomes on a couple, irrespective of whether interest focuses on the entire profile or rather on a specific response at a specific time point (e.g., one partner's outcome in a 
particular year). In the latter case, inference is based using the appropriate subset of parameters from the full longitudinal model.

View 2. One states the scientific question in terms of the outcome at a well-defined point in time, and builds the model accordingly, ignoring all other information available on a couple.

Suppose we are interested in a difference at the last measurement time. Under View 1, we would consider the parameter(s) referring to the last time, while under View 2, we would merely model the outcomes at the last time. While these two seem related and in fact are strongly related for complete data, there are important differences when missingness occurs.

In both cases, we need to reflect on how to deal with missingness. Under View 1, both simple methods, like a complete case analysis, as well as the more advanced direct-likelihood method are possible. Under View 2, since other outcomes on the sample couple are ignored, one cannot make use of such additional information. Arguably, this is not optimal.

Method for handling missingness. A choice has to be made regarding the modeling approach for the missingness process. Under certain assumptions this process can be ignored (e.g., a likelihood-based ignorable analysis or direct-likelihood analysis). Some simple methods, such as a complete case, do not explicitly address the missingness process.

We first describe the measurement and missingness models in turn, then formally introduce and comment on ignorability.

The measurement model will depend on whether or not a full longitudinal analysis is done. 
When the focus is on the last observed measurement or on the last measurement occasion only, one typically opts for classical two- or multi-group comparisons ( $t$ test, Wilcoxon, etc.). When a longitudinal analysis is deemed necessary, the choice depends on the nature of the outcome. For continuous outcomes, one typically assumes a multivariate normal, a special case of the linear mixed-effects model (Verbeke and Molenberghs, 2000):

$$
\boldsymbol{Y}_{i}=X_{i} \boldsymbol{\beta}+\boldsymbol{\varepsilon}_{i}
$$

where $\boldsymbol{Y}_{i}$ is the $n$-dimensional response vector for subject $i, 1 \leq i \leq N, N$ is the number of subjects, $X_{i}$ is a $(n \times p)$ known design matrix, $\boldsymbol{\beta}$ is the $p$ dimensional vector containing the fixed effects, $\varepsilon_{i} \sim N\left(\mathbf{0}, \Sigma_{i}\right)$, with $\Sigma_{i}$ a general or structured variance covariance matrix.

Assume that incompleteness is due to dropout only, and that the first measurement $Y_{i 1}$ is obtained for everyone. A possible model for the dropout process is a logistic regression for the probability of dropout at occasion $j$, given that the subject is still in the study. We denote this probability by $g\left(\boldsymbol{h}_{i j}, y_{i j}\right)$ in which $\boldsymbol{h}_{i j}$ is a vector containing all responses observed up to but not including occasion $j$, as well as relevant covariates. It is assumed that the covariates in the measurement and dropout models are chosen such that the posited missing data mechanism holds. We then assume that $g\left(\boldsymbol{h}_{i j}, y_{i j}\right)$ satisfies

$$
\operatorname{logit}\left[g\left(\boldsymbol{h}_{i j}, y_{i j}\right)\right]=\operatorname{logit}\left[\operatorname{pr}\left(D_{i}=j \mid D_{i} \geq j, \boldsymbol{y}_{i}, \boldsymbol{h}_{i j}\right)\right]=\boldsymbol{h}_{i j} \boldsymbol{\psi}+\omega y_{i j}, \quad i=1, \ldots, N
$$

(Diggle and Kenward, 1994). Thus, the probability to leave the study is allowed to depend on the observed past but is not dependent on, say, current marital satisfaction given the observed past. In principle, it is even allowed for the data of one partner only to be missing. When $\omega$ equals zero, the dropout model is MAR, and all parameters can be estimated using standard software since the measurement model, for which we use a linear mixed model, and the dropout model, assumed to follow a logistic regression, can then be 
fitted separately. If $\omega \neq 0$, the posited dropout process is MNAR (missing not at random), where missingness is allowed to depend on both observed and unobserved outcomes. Model (3.2) provides the building blocks for the dropout process $f\left(d_{i} \mid \boldsymbol{y}_{i}, \boldsymbol{\psi}\right)$.

Rubin (1976) and Little and Rubin (1987) have shown that, under MAR and the condition that the parameters $\boldsymbol{\theta}$ and $\boldsymbol{\psi}$ are functionally independent, likelihood-based inference remains valid when the missing data mechanism is ignored (Verbeke and Molenberghs, 2000, see also ). Practically speaking, the likelihood of interest is then based upon the factor $f\left(\boldsymbol{y}_{i}^{o} \mid \boldsymbol{\theta}\right)$. This is called ignorability. The practical implication is that a software module with likelihood estimation facilities and with the ability to handle incompletely observed subjects, manipulates the correct likelihood, providing valid parameter estimates and likelihood ratio values if the measurement model for the joint outcome distribution holds. Note that the estimands are the parameters of model (3.1), which is a model for complete data, corresponding to what one would expect to see in the absence of dropouts. A similar method is the full information maximum likelihood (FIML) method (Wothke, 2000), which maximizes the likelihood of the observed data in terms of the information available for each parameter. This method has already been used within the sociological research.

Note that an MNAR analysis can proceed only under View 1, i.e., a full longitudinal analysis is necessary, even when interest lies, for example, in a comparison between the answer of both partners at the last occasion. In the latter case, the fitted model can be used as the basis for inference at the last occasion. A common criticism is that a model needs to be considered, with the risk of model misspecification. However, it should be noted that in many sociological settings the repeated measures are balanced in the sense that a common (and often limited) set of measurement times is considered for all subjects, 
allowing the a priori specification of a saturated regression model (e.g., a full group by time interaction model for the fixed effects and unstructured variance-covariance matrix). Of course, such a model is still based on distributional assumptions, such as normality and hence the data analyst may want to consider appropriate forms of sensitivity analysis (Verbeke and Molenberghs, 2000). Nevertheless, an approach of this type is a promising alternative to the often used simple methods such as complete-case analysis. The complete-case and direct-likelihood methods will be described in the next section and studied further in subsequent sections.

\section{Simple Methods and Direct Likelihood}

We will review the commonly used but criticized complete case analysis, for which MCAR is required. Next to these, we will comment on so-called imputation strategies, where data are filled in. With these, a single value is substituted for every "hole" in the data set and the resulting data set is analyzed as if it represented the true complete data.

A complete case analysis includes only those cases for which all measurements were recorded. This method has obvious advantages. It is simple to describe and almost any software can be used since there are no missing data. Unfortunately, the method suffers from severe drawbacks. Even small amounts of missingness on each of the measurement occasions can result in an overal proportion of complete cases which is unacceptably low. Further, severe bias can result when the missingness mechanism is MAR but not MCAR. Indeed, should an estimator be consistent in the complete data problem, then the derived complete case analysis is consistent only if the missingness process is MCAR.

An alternative way to obtain a data set on which complete data methods can be used is to 
fill in rather than delete (Little and Rubin, 1987). Concern has been raised regarding single imputation strategies. Dempster and Rubin (1983) write: "The idea of imputation is both seductive and dangerous. It is seductive because it can lull the user into the pleasurable state of believing that the data are complete after all, and it is dangerous because it lumps together situations where the problem is sufficiently minor that it can be legitimately handled in this way and situations where standard estimators applied to the real and imputed data have substantial biases." It is generally not acceptable for hierarchical models and multivariate models.

Thus, the user of imputation strategies faces several dangers. First, the imputation model could be wrong and, hence, the point estimates biased. Second, even for a correct imputation model, the uncertainty resulting from missingness is ignored. Indeed, even when one is reasonably sure about the mean value the unknown observation would have had, the actual stochastic realization, depending on both the mean and error structures, is still unknown. In addition, most methods require the MCAR assumption to hold while some even require additional and often unrealistically strong assumptions.

\section{On the other hand, a likelihood-based ignorable analysis or direct-likelihood} method produces expectations for the missing observations but no explicit imputation takes place, hence the amount of information in the data is not overestimated and important model elements, such as mean structure and variance components, are not distorted.

Historically, an important motivation behind the simpler methods was their very simplicity. Currently, with the availability of commercial software tools such as, for example, the SAS procedure MIXED, this motivation no longer applies. Arguably, an MAR analysis is the 
preferred choice. Of course, the correctness of an MAR analysis rests upon the truth of the MAR assumption, which is, in turn, never completely verifiable. Note that purely resorting to MNAR analyses is not satisfactory either, since such models are known to be sensitive to unverifiable model assumptions that necessarily have to be made. Indeed, different assumptions imply different fits to the unobserved data, given the observed ones, a part of the model that is by definition not verifiable from the data. The issue is compounded when one tries to estimate such parameters as $\omega$. These and related issues are discussed in Verbeke and Molenberghs (2000) and Molenberghs et al. (2004).

\section{Analysis of Case Studies}

The analyses are performed using the SAS procedure MIXED, allowing the data to have repeated measures for each partner within a couple, and specifying a certain covariance between those repeated measures. While the differences between the fits for the covariance models are not tremendous, it is advisable in situations like the ones considered here, with relatively few measurements per independent unit, to consider a fairly general covariance structure, to reduced the impact of misspecification.

Only the couples with complete information for ALL covariates were used in the analyses, to overcome the problem of fitting submodels on different sets of data. Missing covariates were rather infrequent. First, we split the data into two separate sets of data, one only containing the information of the wives, the other with only the information of the husbands. Both data sets were analyzed separately, ignoring the possible correlation between husband's and wife's responses. Afterwards, the analyses were redone on the partners simultaneously, allowing us to also model the correlation between the partners' responses. In both cases, we first restricted the analyses to the couples with observations in 
1990 as well as in 1995, the so-called complete case (CC) analysis. Secondly, the data were analyzed as they are (for some couples both 1990 and 1995, for others only 1990), consistent with ignorability, and therefore this will be further called the direct-likelihood (DL) analysis.

All analyses were repeated for the 3 outcomes of interest, namely marital satisfaction, open communication and negative communication.

\subsection{Separate Analyses for Husband and Wife}

First of all, a separate analysis is performed for husbands and wives, to easily find gender specific covariates that might influence the outcomes of interest. Since our interest lies in a lot of covariate effects, and the model would become too extensive if all effects, together with their interactions with year of questionnaire, are included at once, we started the analyses with a single covariate selection, including the covariate itself, year, and the interaction effect, to allow the effect of this covariate to change over time. After selecting all significant effects from this single covariate approach, we combined them into a new model, from which we started removing the non-significant effects again. Finally, when all remaining covariates are (borderline) significant, the covariance matrix, which is kept unstructured until now, will be reduced. This matrix contains the covariances between the observations in 1990 and 1995. Therefore, it has to be a $2 \times 2$ matrix, for which only a few covariance structures are possible, namely unstructured (UN), banded main diagonal (UN(1)), compound symmetry (CS), and simple (SIMPLE), respectively, as shown below:

$$
\left(\begin{array}{cc}
\sigma_{1}^{2} & \sigma_{12} \\
\sigma_{12} & \sigma_{2}^{2}
\end{array}\right) \quad\left(\begin{array}{cc}
\sigma_{1}^{2} & 0 \\
0 & \sigma_{2}^{2}
\end{array}\right) \quad\left(\begin{array}{cc}
\sigma^{2}+\sigma_{1}^{2} & \sigma_{1}^{2} \\
\sigma_{1}^{2} & \sigma^{2}+\sigma_{1}^{2}
\end{array}\right) \quad\left(\begin{array}{cc}
\sigma^{2} & 0 \\
0 & \sigma^{2}
\end{array}\right)
$$

Results from the analyses for marital satisfaction, open and negative communication are 
shown in Tables $2-4$, respectively. For the 3 outcomes, we see that all significant covariate effects in the CC model remain significant in the final model for the DL analysis, unless they were borderline significant in the CC case, then they turned out to be non-significant in the DL analysis. In the latter, sometimes even more covariate effects appeared to be significant, and the significance becomes stronger for almost all covariates.

Table 2 about here

Table 3 about here

Table 4 about here

Marital satisfaction increases between 1990 and 1995, both for males and females. For the husbands, education has a borderline significant decreasing effect on marital satisfaction when only considering the completers. For the wives, year of birth has a changing effect over time in the $\mathrm{CC}$ analysis, while a constant increasing effect in the DL analysis. There is a positive correlation between the 1990 and 1995 marital satisfaction of about 0.61 .

Open communication increases between 1990 and 1995 for the males, but decreases for the females. For the husbands, in both $\mathrm{CC}$ and DL analysis, year of marriage has a borderline significant decreasing effect on open communication, and year of birth a changing effect over time. In the DL analysis, there is an additional changing effect of income over time. 
For the wives, in both CC and DL analysis, income has a changing effect over time on open communication, and year of birth a constant increasing effect. There is a positive correlation between the 1990 and 1995 open communication of about 0.41 for the males, and about 0.48 for the females.

Negative communication does not change between 1990 and 1995 for the males, and decreases for the females. For the husbands, there is only a borderline significant increasing effect of marital status on negative communication, in the $\mathrm{CC}$ analysis. For the wives, in both CC and DL analysis, the number of children and the year of marriage have a changing effect over time on negative communication. In the DL analysis, there is an additional changing effect of income over time. There is a positive correlation between the 1990 and 1995 negative communication of about 0.61 .

\subsection{Joint Analysis for Both Partners}

When analyzing both partners at the same time, in one model, we can still allow the covariates to have a different effect on the outcome for both partners. Therefore not only the interaction with year, but also with partner will be included in the model, and even higher order interactions of the form covariate $\times$ year $\times$ partner will be included. Since this results in even more dependent variables in the model, we will explore the variables of interest one by one, together with all relevant interaction terms. Afterwards, we will again remove the non-significant terms which remained after combining all significant effects from the single covariate selection, and reduce the covariance matrix. Since we are now not only interested in possible correlations between the years of questionnaire, but also in possible correlations between the partners, the covariance matrices in (5.3) should be extended to $4 \times 4$ matrices. These matrices are of the most general form possible and require in the 
unstructured cases 10 parameters to be estimated. Therefore, we will immediately reduce the covariance matrix to the Kronecker product of an unstructured matrix, modeling the covariance across the multivariate observations (partners) with an additional covariance matrix (unstructured, compound symmetry, or first-order autoregressive $(\mathrm{AR}(1))$ ), modeling the covariance across time. The upper left value in the second matrix is constrained to equal 1 to identify the model. This Kronecker product also results in a $4 \times 4$ matrix, but with less parameters to estimate. Shown below are the UN@UN and UN@CS type, respectively. Since we only consider 2 measurement occasion, UN@AR(1) is identical to UN@CS.

$$
\begin{aligned}
\left(\begin{array}{cc}
\sigma_{1}^{2} & \sigma_{12} \\
\sigma_{12} & \sigma_{2}^{2}
\end{array}\right) \otimes\left(\begin{array}{cc}
1 & \sigma_{23} \\
\sigma_{23} & \sigma_{4}^{2}
\end{array}\right) & =\left(\begin{array}{cccc}
\sigma_{1}^{2} & \sigma_{1}^{2} \sigma_{23} & \sigma_{12} & \sigma_{12} \sigma_{23} \\
\sigma_{1}^{2} \sigma_{23} & \sigma_{1}^{2} \sigma_{4}^{2} & \sigma_{12} \sigma_{23} & \sigma_{12} \sigma_{4}^{2} \\
\sigma_{12} & \sigma_{12} \sigma_{23} & \sigma_{2}^{2} & \sigma_{2}^{2} \sigma_{23} \\
\sigma_{12} \sigma_{23} & \sigma_{12} \sigma_{4}^{2} & \sigma_{2}^{2} \sigma_{23} & \sigma_{2}^{2} \sigma_{4}^{2}
\end{array}\right) \\
\left(\begin{array}{cc}
\sigma_{1}^{2} & \sigma_{12} \\
\sigma_{12} & \sigma_{2}^{2}
\end{array}\right) \otimes\left(\begin{array}{ll}
1 & \rho \\
\rho & 1
\end{array}\right) & =\left(\begin{array}{cccc}
\sigma_{1}^{2} & \sigma_{1}^{2} \rho & \sigma_{12} & \sigma_{12} \rho \\
\sigma_{1}^{2} \rho & \sigma_{1}^{2} & \sigma_{12} \rho & \sigma_{12} \\
\sigma_{12} & \sigma_{12} \rho & \sigma_{2}^{2} & \sigma_{2}^{2} \rho \\
\sigma_{12} \rho & \sigma_{12} & \sigma_{2}^{2} \rho & \sigma_{2}^{2}
\end{array}\right)
\end{aligned}
$$

To use these structures in the SAS procedure MIXED, it is necessary to specify two distinct REPEATED effects, e.g. partner and year, both included in the CLASS statement, and in the same order as they appear in the Kronecker product.

Results from the analyses for marital satisfaction, open and negative communication are shown in Tables 5-7, respectively.

Table 5 about here 
Table 6 about here

Table 7 about here

In general, the same significant covariate effects appear as in the separate analyses for husband and wife. If a certain covariate was significant for both partners, then this effect is present in the same way as before, only the main effect, or also the interaction with year. If, on the other hand, the covariate was only significant for one of both partners, then now this effect, and a possible interaction with year, is present in the model, together with its interaction with partner. Some covariates disappear from the model, but this was restricted to those that were borderline significant when analyzing the partners separately. So we can say that both ways of analyses give comparable results.

The simultaneous modeling of the partners allows us to have an idea about the baseline outcomes, i.e., the corrected effect after taking covariate information into account, for husband and wife, both in 1990 and 1995, and to decide whether they significantly differ from each other. We also get a handle on the association between them. We can draw the following conclusions for the 3 outcomes of interest. The marital satisfaction for wife is lower than that of husband in 1990, and both increase in 1995. In the DL analysis, this increase is similar for both partners, while in the $\mathrm{CC}$ analysis, the increase for wife is much higher than for husband. The open communication is higher for husband than for wife. The evolution over time is the same for both partners, but is of a decreasing trend in the CC analysis, while of an increasing trend in the DL analysis. Finally, the conclusions for 
last outcome of interest are much more complicated. In the CC analysis, negative communication decreases over time, but only marginally so for the husband, and much more for the wife, while in the DL analysis, negative communication increases a bit for males, and decreases for females. So in summary, we can say that negative communication approximately stays the same for the husband, and decreases considerably for the wife. In both ways of analysis, the wife has a higher value than the husband in 1990, but a lower in 1995.

The covariance components in Tables 5-7 allow us to get an idea of the within-partner correlation, as well as between-partner correlation. For example, from Table 6 we observe the within-partner correlation is around 0.40, while between-partner it is around 0.30 .

\section{Conclusions and Discussion}

In this paper, we showed that there is little justification for analyzing incomplete marriage satisfaction data, and similar data in sociology, by means of a MCAR method, such as CC.

Although at first sight, the results from CC and direct-likelihood as presented here, do not differ regarding the importance of the predictor variables, the significance as well as non-significance of the effects are much more pronounced in the DL analysis. It is possible that in other studies there are more extreme differences between these types of analyses (Molenberghs et al., 2004), and therefore simple but potentially highly restrictive modes of analyses, such as CC, should no longer be seen as the preferred mode of analysis.

Importantly, we have to note that our conclusions differ somewhat from those often found in the literature. For example, a higher score for males on positive communication is 
somewhat counterintuitive (Canary et al., 2002; Rubin, 1983; Wood, 1993; Weigel and Ballard-Reisch, 2001), as well as an increase in marital satisfaction between 1990 and 1995 (Vaillant and Vaillant, 1993; Van Laningham et al., 2001). Of course, this is at the heart of our proposed methodology, which not only takes the multivariate and longitudinal nature of the data into account, it also properly incorporates information from incomplete records. These results underscore that the traditional modes of analysis, in particular CC, may be too restrictive and even lead to false intuition, since for it to be valid the completers have to be a perfectly random subset of the entire set of data. In contrast, our analysis is valid under the much more flexible MAR assumption, which merely assumes that there is sufficient information on the missingness process in the (partially) observed data. Thus, it is better to use linear mixed models in combination with the assumption of MAR. Moreover, such analyses can be conducted routinely using standard statistical software such as the SAS procedure MIXED.

Since the covariates in our models are relatively highly correlated, one might be inclined to center these variables prior to including them into the models. However, duplication of our analyses with centered covariates led to equivalent results.

Whether our more generally valid analysis would consistently provide differences with those found in the literature is an interesting subject of further study. Of course, it is still possible for the MAR assumption to be violated in favor of MNAR. However, the type of direct-likelihood analysis proposed here often still provides sensible assessments of important aspects of the data, even if the assumption of MAR is violated in favor of MNAR. Nevertheless, it is also possible to explore the effect of violation of MAR by conducting appropriate forms of sensitivity analysis (Verbeke et al., 2001; Van Steen et al., 2001; Molenberghs et al., 2001; Jansen et al., 2003). 


\section{Acknowledgments}

Ivy Jansen and Geert Molenberghs gratefully acknowledge support from

Fonds Wetenschappelijk Onderzoek-Vlaanderen Research Project G.0002.98 "Sensitivity Analysis for Incomplete and Coarse Data" and from Belgian IUAP/PAI network "Statistical Techniques and Modeling for Complex Substantive Questions with Complex Data". 


\section{References}

Amato, Paul R. 1996. "Explaining the Intergenerational Transmission of Divorce." Journal of Marriage and the Family 58:628-640.

Canary, Daniel J., Laura Stafford, and Beth A. Semic. 2002. "A Panel Study of the Associations between Maintenance Strategies and Relational Characteristics." Journal of Marriage and the Family 64:395-406.

Dempster, Arthur P. and Donald B. Rubin. 1983. "Overview." In Incomplete Data in Sample Surveys, Vol. II: Theory and Annotated Bibliography, edited by W. G. Madow, I. Olkin, and D. B. Rubin, pp. 3-10. New York: Academic Press.

Diggle, Peter J. and Mike G. Kenward. 1994. "Informative dropout in longitudinal data analysis (with discussion)." Applied Statistics 43:49-93.

Gerris, Jan R. M., Tilly M. J. M. Houtmans, Elma M. G. Kwaaitaal-Roosen, J. Clasien Schipper, Ad A. Vermulst, and Jan M. A. M. Janssens. 1998. Parents, adolescents and young adults in Dutch families. A longitudinal study. Nijmegen: University of Nijmegen, Institute of Family Studies.

Gerris, Jan R. M., Dorien A. A. M. Van Boxtel, Ad A. Vermulst, Jan M. A. M. Janssens, René A. H. Van Zutphen, and Albert J. A. Felling. 1992. Child-rearing, family relations and family processes in 1990. Nijmegen: University of Nijmegen, Institute of Family Studies.

Gerris, Jan R. M., Ad A. Vermulst, Dorien A. A. M. Van Boxtel, Jan M. A. M. Janssens, René A. H. Van Zutphen, and Albert J. A. Felling. 1993. Parenting in Dutch families. Nijmegen: University of Nijmegen, Institute of Family Studies. 
Holman, Thomas B. 2001. Premarital prediction of marital quality or breakup. Research, theory and practice. New York: Kluwer Academic/Plenum Publishers.

Jansen, Ivy, Caroline Beunckens, Geert Molenberghs, Geert Verbeke, and Craig Mallinckrodt. 2005. "Analyzing Incomplete Discrete Longitudinal Clinical Trial Data." Statistical Science Accepted.

Jansen, Ivy, Geert Molenberghs, Marc Aerts, Herbert Thijs, and Kristel Van Steen. 2003. "A local influence approach applied to binary data from a psychiatric study." Biometrics 59:409-418.

Johnson, David R., Teodora O. Amoloza, and Alan Booth. 1992. "Stability and Developmental Change in Marital Quality: A Three-Wave Panel Analysis." Journal of Marriage and the Family 54:582-594.

Karney, Benjamin R. and Thomas N. Bradbury. 1995. "The longitudinal course of marital quality and stability: A review of theory, method and research." Psychological Bulletin 118:3-34.

Lavee, Yoav, Shlomo Sharlin, and Ruth Katz. 1996. "The Effect of Parenting Stress on Marital Quality: An Integrated Mother-Father Model." Journal of Family Issues $17: 114-135$.

Lewis, Robert A. and Graham B. Spanier. 1979. "Theorizing about the quality and stability of marriage." In Contemporary theories about the family: research-base theories., edited by W. R. Burr, R. Hill, F. I. Nye, and I. L. Reiss, pp. 49-65. New York: Free Press.

Little, Roderick J. A. 1982. "Models for nonresponse in sample surveys." Journal of the American Statistical Association 77:237-250. 
Little, Roderick J. A. 1995. "Modeling the drop-out mechanism in repeated-measures studies." Journal of the American Statistical Association 90:1112-1121.

Little, Roderick J. A. and Donald B. Rubin. 1987. Statistical Analysis with Missing Data. New York: Wiley.

Little, Roderick J. A. and Donald B. Rubin. 2002. Statistical Analysis with Missing Data. New York: Wiley.

Molenberghs, Geert, Mike G. Kenward, and Els Goetghebeur. 2001. "Sensitivity analysis for incomplete contingency tables: The Slovenian plebiscite case." Applied Statistics $50: 15-29$.

Molenberghs, Geert, Herbert Thijs, Ivy Jansen, Caroline Beunckens, Mike G. Kenward, Craig Mallinckrodt, and Raymond J. Carroll. 2004. "Analyzing incomplete longitudinal clinical trial data." Biostatistics 5:445-464.

Rogers, Stacy J. and Lynn K. White. 1998. "Satisfaction with Parenting: The Role of Marital Happiness, Family Structure, and Parents' Gender." Journal of Marriage and the Family 60:293-308.

Rubin, Donald B. 1976. "Inference and missing data." Biometrika 63:581-592. With comments by R. J. A. Little and a reply by the author.

Rubin, Lillian B. 1983. Intimate Strangers: Men and Women Together. New York: Harper \& Row.

Vaillant, Camille O. and George E. Vaillant. 1993. "Is the U-Curve of Marital Satisfaction an Illusion? A 40-Year Study of Marriage." Journal of Marriage and the Family $55: 230-239$. 
Van den Troost, Ann, Ad A. Vermulst, Jan R. M. Gerris, and Koenraad Matthijs. 2001. "Meetinvariantie van huwelijkskwaliteit en satisfactie." Leuven/Nijmegen. Onderzoeksverslag van het Departement Sociologie - afdeling Gezin, Bevolking \& Gezondheidszorg en Orthopedagogiek. - Gezin en Gedrag GB/2001-13.

Van Laningham, Jody, David R. Johnson, and Paul Amato. 2001. "Marital Happiness, Marital Duration, and the U-Shaped Curve: Evidence from a Five-Wave Panel Study." Social Forces 79:1313-1341.

Van Steen, Kristel, Geert Molenberghs, Geert Verbeke, and Herbert Thijs. 2001. "A local influence approach to sensitivity analysis of incomplete longitudinal ordinal data." Statistical Modelling: An International Journal 1:125-142.

Verbeke, Geert and Geert Molenberghs. 2000. Linear Mixed Models for Longitudinal Data. New York: Springer-Verlag.

Verbeke, Geert, Geert Molenberghs, Herbert Thijs, Emmanuel Lesaffre, and Mike G. Kenward. 2001. "Sensitivity analysis for non-random dropout: A local influence approach." Biometrics 57:7-14.

Weigel, Daniel J. and Deborah S. Ballard-Reisch. 2001. "The impact of relational maintenance behaviors on marital satisfaction: A longitudinal analysis." Journal of Family Communication 1:265-279.

Wood, Julia T. 1993. "Engendered relations: Interaction, caring, power and responsibility in intimacy." In Social context and social relationships., edited by S. Duck, pp. 26-54. Newbury Park/London: Sage Publications.

Wothke, Werner. 2000. "Longitudinal and multi-group modeling with missing data." In Modeling Longitudinal and Multilevel Data: Practical Issues, Applied Approaches and 
Specific Examples, edited by T. D. Little, K. U. Schnabel, and J. Baumert, pp. 3-10. Mahwah, N.J.: Lawrence Erlbaum. 
Table 1: Reasons why families refused to participate in 1990 and 1995.

\begin{tabular}{|l|c|c|}
\hline \multicolumn{1}{|c|}{ Reasons to refuse } & Number of families \\
\cline { 2 - 3 } & in $\mathbf{1 9 9 0}$ & in 1995 \\
\hline Family was not interested or no time & 761 & 72 \\
Family was approached three times without success & 79 & 10 \\
Address is unknown or not correct & 43 & 6 \\
Respondent was died, ill or on holiday & 32 & 7 \\
The child was too young or too old for being eligible & 22 & 0 \\
Linguistic problem & 76 & 1 \\
Invasion of privacy & 0 & 7 \\
Couple was divorced or problems in the family & 0 & 8 \\
Prior research was too long ago & 0 & 9 \\
Otherwise & 28 & 23 \\
\hline Total & $\mathbf{1 0 4 1}$ & $\mathbf{1 4 3}$ \\
\hline Source: Geris at (1992 & & \\
\hline
\end{tabular}

Source: Gerris et al $(1992 ; 1998)$ 
Table 2: MARITAL SATISFACTION. Remaining (borderline) significant effects. Year equals 1 for 1990, 0 for 1995. Marstat equals 1 if married, 0 if divorced.

\begin{tabular}{|c|c|c|c|c|c|c|c|c|c|c|c|c|}
\hline & \multicolumn{6}{|c|}{ FATHER } & \multicolumn{6}{|c|}{ MOTHER } \\
\hline & \multicolumn{3}{|c|}{$\begin{array}{c}\text { CC } \\
(294 \text { subj. / } 588 \text { obs. })\end{array}$} & \multicolumn{3}{|c|}{$\begin{array}{c}\text { DL } \\
\text { (603 subj. / } 893 \text { obs.) }\end{array}$} & \multicolumn{3}{|c|}{$\begin{array}{c}\text { CC } \\
(294 \text { subj. / } 588 \text { obs. })\end{array}$} & \multicolumn{3}{|c|}{$\begin{array}{c}\text { DL } \\
\text { (608 subj. / } 931 \text { obs.) }\end{array}$} \\
\hline & est & s.e. & $p$-value & est & s.e. & $p$-value & est & s.e. & $p$-value & est & s.e. & $p$-value \\
\hline intercept & 6.1826 & 0.1060 & $<.0001$ & 6.1982 & 0.0819 & $<.0001$ & 6.6832 & 0.7317 & $<.0001$ & 4.7177 & 0.4492 & $<.0001$ \\
\hline year & -0.7785 & 0.0473 & $<.0001$ & -0.7870 & 0.0455 & $<.0001$ & -2.1303 & 0.6116 & 0.0006 & -0.7350 & 0.0490 & $<.0001$ \\
\hline education & -0.0400 & 0.0230 & 0.0831 & -0.0587 & 0.0170 & 0.0006 & - & - & - & - & - & - \\
\hline birthyear & - & - & - & - & - & - & -0.0136 & 0.0146 & 0.3524 & 0.0238 & 0.0089 & 0.0078 \\
\hline birthyear $\times$ year & - & - & - & - & - & - & 0.0270 & 0.0122 & 0.0273 & - & - & - \\
\hline \multirow{2}{*}{$\begin{array}{l}\text { covariance structure } \\
\text { covariance matrix }\end{array}$} & \multicolumn{3}{|c|}{ UN } & \multirow{2}{*}{\multicolumn{3}{|c|}{$\begin{array}{c}\text { UN } \\
\left(\begin{array}{ll}0.7204 & 0.5162 \\
0.5162 & 0.9881\end{array}\right)\end{array}$}} & \multirow{2}{*}{\multicolumn{3}{|c|}{$\begin{array}{c}\text { UN } \\
\left(\begin{array}{ll}0.8437 & 0.5873 \\
0.5873 & 1.0980\end{array}\right)\end{array}$}} & \multicolumn{3}{|c|}{ UN } \\
\hline & \multicolumn{3}{|c|}{$\left(\begin{array}{ll}0.6482 & 0.4652 \\
0.4652 & 0.9405\end{array}\right)$} & & & & & & & \multicolumn{3}{|c|}{$\left(\begin{array}{ll}0.8739 & 0.6290 \\
0.6290 & 1.2295\end{array}\right)$} \\
\hline$-2 \ell$ & \multicolumn{3}{|c|}{1394.2} & \multicolumn{3}{|c|}{2195.8} & \multicolumn{3}{|c|}{1509.2} & \multicolumn{3}{|c|}{2478.4} \\
\hline
\end{tabular}


Table 3: OPEN COMMUNICATION. Remaining (borderline) significant effects. Year equals 1 for 1990, 0 for 1995. Marstat equals 1 if married, 0 if divorced.

\begin{tabular}{|c|c|c|c|c|c|c|c|c|c|c|c|c|}
\hline & \multicolumn{6}{|c|}{ FATHER } & \multicolumn{6}{|c|}{ MOTHER } \\
\hline & \multicolumn{3}{|c|}{$\begin{array}{c}\text { CC } \\
(294 \text { subj. / } 588 \text { obs. })\end{array}$} & \multicolumn{3}{|c|}{$\begin{array}{c}\text { DL } \\
\text { (603 subj. / } 895 \text { obs.) }\end{array}$} & \multicolumn{3}{|c|}{$\begin{array}{c}\text { CC } \\
\text { (294 subj. / } 588 \text { obs. })\end{array}$} & \multicolumn{3}{|c|}{$\begin{array}{c}\text { DL } \\
\text { (608 subj. / } 934 \text { obs. })\end{array}$} \\
\hline & est & s.e. & $p$-value & est & s.e. & $p$-value & est & s.e. & $p$-value & est & s.e. & $p$-value \\
\hline intercept & 8.0458 & 1.2288 & $<.0001$ & 6.7478 & 1.0662 & $<.0001$ & 3.1474 & 0.7571 & $<.0001$ & 3.1285 & 0.5839 & $<.0001$ \\
\hline year & -1.6311 & 0.6665 & 0.0150 & -0.9555 & 0.7208 & 0.1860 & 1.0065 & 0.3522 & 0.0046 & 0.7704 & 0.2975 & 0.0100 \\
\hline maryear & -0.0371 & 0.0200 & 0.0640 & -0.0327 & 0.0156 & 0.0364 & - & - & - & - & - & - \\
\hline income & - & - & - & 0.0638 & 0.0470 & 0.1758 & 0.1172 & 0.0488 & 0.0170 & 0.1051 & 0.0412 & 0.0112 \\
\hline income $\times$ year & - & - & - & -0.1169 & 0.0514 & 0.0238 & -0.1721 & 0.0524 & 0.0011 & -0.1395 & 0.0444 & 0.0018 \\
\hline birthyear & -0.0035 & 0.0152 & 0.8184 & 0.0074 & 0.0136 & 0.5872 & 0.0347 & 0.0128 & 0.0069 & 0.0364 & 0.0097 & 0.0002 \\
\hline birthyear $\times$ year & 0.0324 & 0.0140 & 0.0209 & 0.0340 & 0.0127 & 0.0078 & - & - & - & - & - & - \\
\hline \multirow[t]{2}{*}{ covariance structure } & \multicolumn{3}{|c|}{ CS } & \multicolumn{3}{|c|}{ CS } & \multicolumn{3}{|c|}{$\mathrm{CS}$} & \multicolumn{3}{|c|}{$\mathrm{CS}$} \\
\hline & \multicolumn{3}{|c|}{$\left(\begin{array}{ll}1.2134 & 0.5000 \\
0.5000 & 1.2134\end{array}\right)$} & \multicolumn{3}{|c|}{$\left(\begin{array}{ll}1.2334 & 0.4957 \\
0.4957 & 1.2334\end{array}\right)$} & \multicolumn{3}{|c|}{$\left(\begin{array}{ll}1.1212 & 0.5271 \\
0.5271 & 1.1212\end{array}\right)$} & \multicolumn{3}{|c|}{$\left(\begin{array}{ll}1.1591 & 0.5580 \\
0.5580 & 1.1591\end{array}\right)$} \\
\hline$-2 \ell$ & \multicolumn{3}{|c|}{1727.7} & \multicolumn{3}{|c|}{2674.3} & \multicolumn{3}{|c|}{1662.5} & \multicolumn{3}{|c|}{2478.4} \\
\hline
\end{tabular}


Table 4: NEGATIVE COMMUNICATION. Remaining (borderline) significant effects. Year equals 1 for 1990, 0 for 1995. Marstat equals 1 if married, 0 if divorced.

\begin{tabular}{|c|c|c|c|c|c|c|c|c|c|c|c|c|}
\hline & \multicolumn{6}{|c|}{ FATHER } & \multicolumn{6}{|c|}{ MOTHER } \\
\hline & \multicolumn{3}{|c|}{$\begin{array}{c}\text { CC } \\
\text { (294 subj. / } 588 \text { obs.) }\end{array}$} & \multicolumn{3}{|c|}{$\begin{array}{c}\text { DL } \\
\text { (608 subj. / } 932 \text { obs.) }\end{array}$} & \multicolumn{3}{|c|}{$\begin{array}{c}\text { CC } \\
\text { (294 subj. / } 588 \text { obs. })\end{array}$} & \multicolumn{3}{|c|}{$\begin{array}{c}\text { DL } \\
(608 \text { subj. / } 932 \text { obs. })\end{array}$} \\
\hline & est & s.e. & $p$-value & est & s.e. & $p$-value & est & s.e. & $p$-value & est & s.e. & $p$-value \\
\hline intercept & 2.4711 & 0.1307 & $<.0001$ & 2.7383 & 0.0401 & $<.0001$ & 2.6771 & 1.2950 & 0.0396 & 2.4958 & 1.1744 & 0.0340 \\
\hline year & - & - & - & - & - & - & 3.9312 & 1.1677 & 0.0009 & 3.6186 & 1.1047 & 0.0012 \\
\hline children & - & - & - & - & - & - & -0.0295 & 0.0467 & 0.5289 & 0.0202 & 0.0429 & 0.6385 \\
\hline children $\times$ year & - & - & - & - & - & - & -0.1113 & 0.0467 & 0.0179 & -0.1080 & 0.0426 & 0.0116 \\
\hline maryear & - & - & - & - & - & - & 0.0007 & 0.0176 & 0.9675 & 0.0019 & 0.0153 & 0.8990 \\
\hline maryear $\times$ year & - & - & - & - & - & - & -0.0502 & 0.0158 & 0.0016 & -0.0402 & 0.0142 & 0.0051 \\
\hline marstat & 0.2811 & 0.1443 & 0.0524 & - & - & - & - & - & - & - & - & - \\
\hline income & & - & - & & & & & & & 0.0051 & 0.0372 & 0.8911 \\
\hline income $\times$ year & - & - & - & - & - & - & - & - & - & -0.0651 & 0.0382 & 0.0897 \\
\hline \multirow{3}{*}{$\begin{array}{l}\text { covariance structure } \\
\text { covariance matrix }\end{array}$} & \multicolumn{3}{|c|}{$\mathrm{CS}$} & \multicolumn{3}{|c|}{$\mathrm{CS}$} & \multicolumn{3}{|c|}{$\mathrm{CS}$} & \multicolumn{3}{|c|}{ CS } \\
\hline & \multirow{2}{*}{\multicolumn{3}{|c|}{$\left(\begin{array}{ll}1.1213 & 0.6889 \\
& \\
0.6889 & 1.1213\end{array}\right)$}} & \multirow{2}{*}{\multicolumn{3}{|c|}{$\left(\begin{array}{ll}1.1032 & 0.6706 \\
& \\
0.6706 & 1.1032\end{array}\right)$}} & \multirow{2}{*}{\multicolumn{3}{|c|}{$\left(\begin{array}{ll}1.0313 & 0.6180 \\
& \\
0.6180 & 1.0313\end{array}\right)$}} & \multirow{2}{*}{\multicolumn{3}{|c|}{$\left(\begin{array}{ll}1.0870 & 0.6754 \\
0.6754 & 1.0870\end{array}\right)$}} \\
\hline & & & & & & & & & & & & \\
\hline$-2 \ell$ & \multicolumn{3}{|c|}{1596.6} & \multicolumn{3}{|c|}{2585.6} & \multicolumn{3}{|c|}{1556.0} & \multicolumn{3}{|c|}{2702.0} \\
\hline
\end{tabular}


Table 5: MARITAL SATISFACTION. Remaining (borderline) significant effects. Parent equals 1 for husband, 0 for wife. Year equals 1 for 1990, 0 for 1995. Marstat equals 1 if married, 0 if divorced.

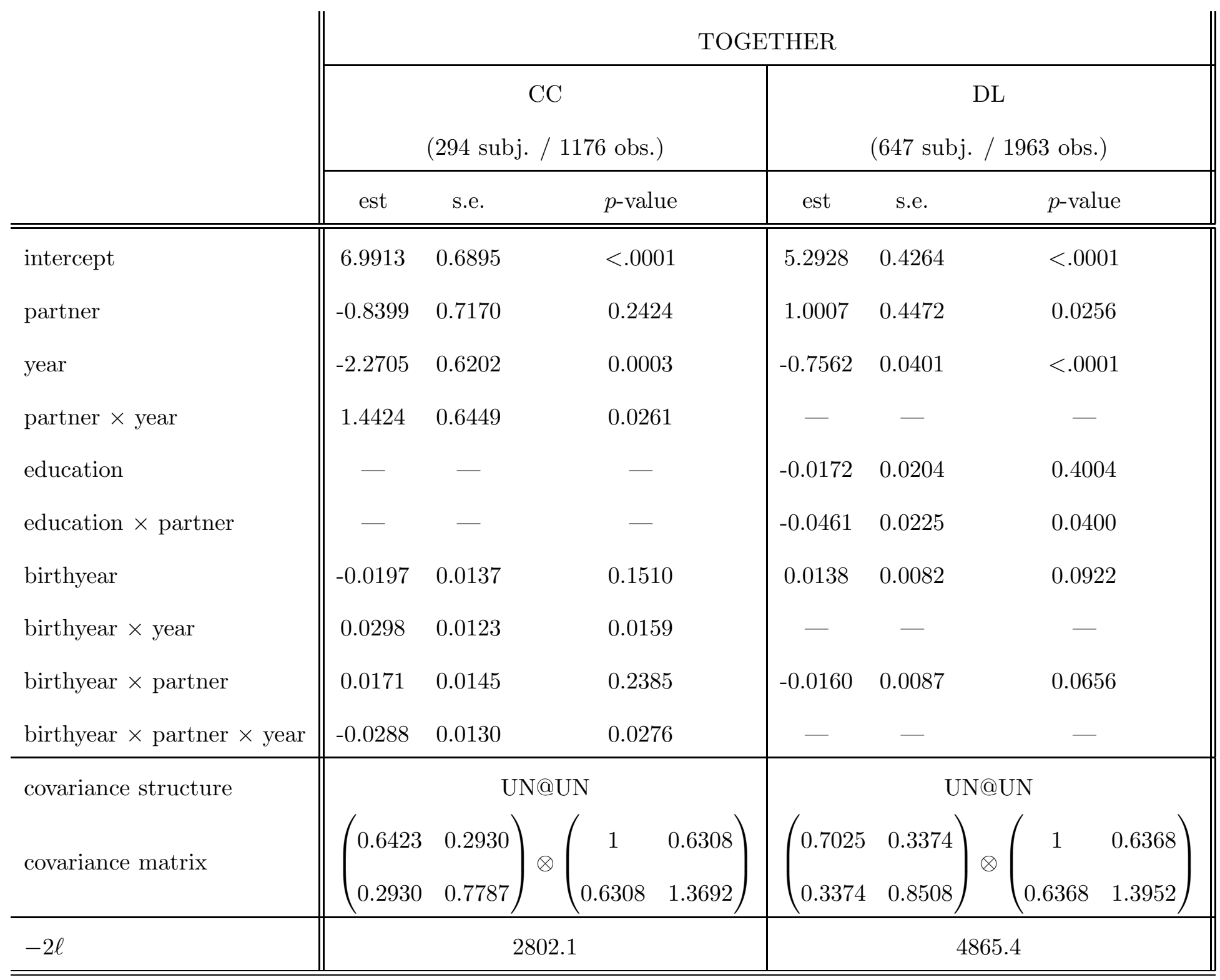


Table 6: OPEN COMMUNICATION. Remaining (borderline) significant effects. Parent equals 1 for husband, 0 for wife. Year equals 1 for 1990, 0 for 1995. Marstat equals 1 if married, 0 if divorced.

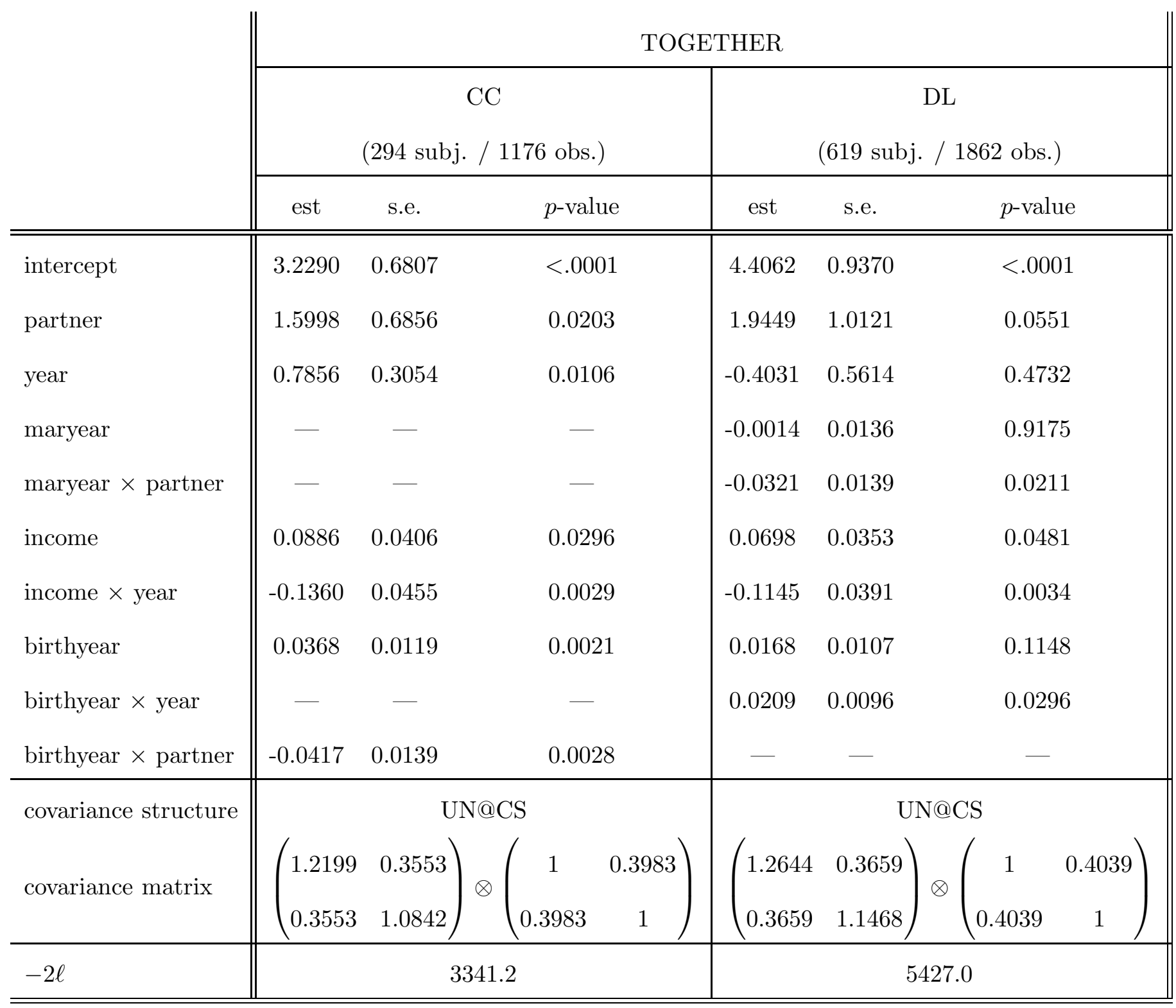


Table 7: NEGATIVE COMMUNICATION. Remaining (borderline) significant effects. Parent equals 1 for husband, 0 for wife. Year equals 1 for 1990, 0 for 1995. Marstat equals 1 if married, 0 if divorced.

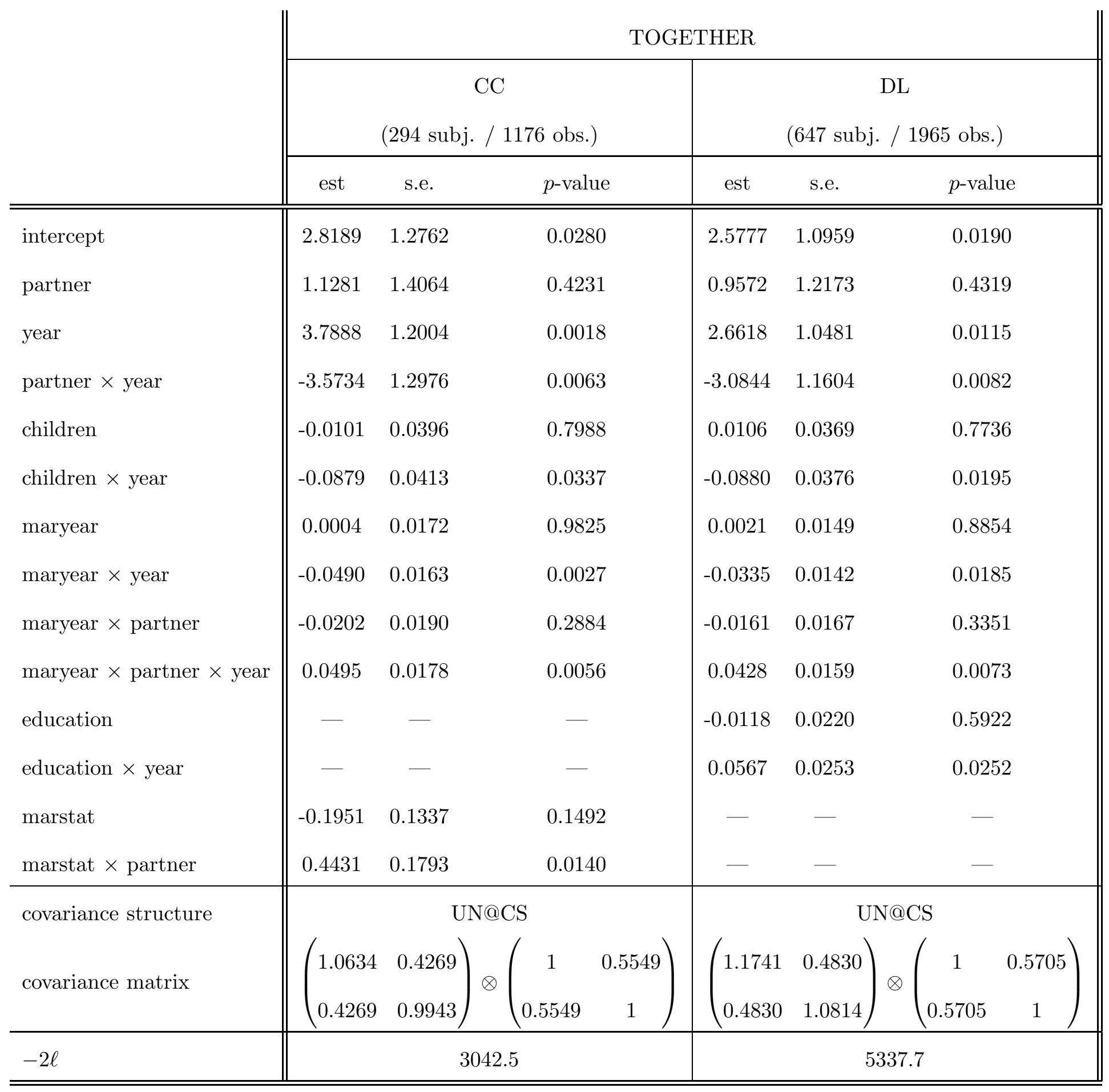

